\title{
Management of Primary Nonresponders and Partial Responders to Tumor Necrosis Factor-a Inhibitor Induction Therapy among Patients with Crohn's Disease
}

\author{
Hideki lijima $^{\text {a }}$ Taku Kobayashi $^{\text {b }}$ Mitsuo Nagasaka ${ }^{c}$ Shinichiro Shinzaki ${ }^{a}$ \\ Kazuya Kitamura $^{d}$ Yasuo Suzuki $^{e}$ Mamoru Watanabe $^{f}$ \\ Toshifumi Hibi $^{\text {b }}$ on behalf of JRT Group \\ a Department of Gastroenterology and Hepatology, Osaka University Graduate School of Medicine, Suita, Japan; \\ ${ }^{b}$ Center for Advanced IBD Research and Treatment, Kitasato University Kitasato Institute Hospital, Tokyo, Japan;

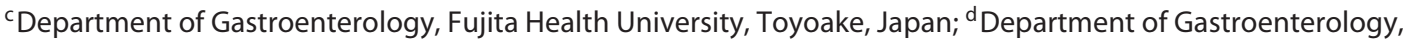

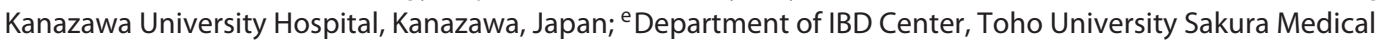 \\ Center, Sakura, Japan; ' Department of Gastroenterology and Hepatology, Tokyo Medical and Dental University, \\ Tokyo, Japan
}

\section{Keywords}

Crohn's disease - Infliximab - Expert opinion · Primary

nonresponder $\cdot$ Partial responder

\begin{abstract}
Induction therapy with tumor necrosis factor-a (TNF-a) inhibitors is highly effective for the treatment of Crohn's disease. However, there are primary nonresponders (PNR) of TNF-a inhibitors without clinical response during the induction period. In addition, there are partial responders (PR), who show some efficacy, but clinical remission is not achieved by induction therapy. To date, the definition and clinical management of PNR and PR have not been established. This report summarizes the opinions of 36 Japanese experts attending the Japan Round Table Discussion on IBD Meeting regarding how to determine PNR and PR of TNF-a inhibitors and how to manage these patients in clinical practice. PNR, who do not show any initial improvement of clini-
\end{abstract}

\begin{tabular}{ll}
\hline KARGER & ( ) 2020 The Author(s) \\
& Published by S. Karger AG, Basel Openger \\
& This article is licensed under the Creative Commons Attribution- \\
karger@karger.com & NonCommercial-NoDerivatives 4.0 International License (CC BY- \\
NC-ND) (http://www.karger.com/Services/OpenAccessLicense). \\
Uswwe and distribution for commercial purposes as well as any dis- \\
tribution of modified material requires written permission.
\end{tabular}

cal symptoms and serum C-reactive protein (CRP) levels, require re-assessment of intestinal complications. PR can be determined either by clinical symptoms, serum CRP levels, or imaging results. PR need intensification of the treatment with TNF-a inhibitors either with or without optimization of immunomodulators. Optimization of initial TNF-a inhibitor therapy may improve long-term outcomes, but more evidence is required to improve the use of TNF-a inhibitors for the prevention and management of PNR and PR.

$$
\begin{aligned}
& \text { (c) } 2020 \text { The Author(s) } \\
& \text { Published by S. Karger AG, Basel }
\end{aligned}
$$

\section{Introduction}

Crohn's disease (CD) is a chronic inflammatory disease of unknown etiology in inflammatory bowel disease (IBD) characterized by transmural granulomatous inflammation in the digestive tract and sometimes complicated by fistulas and abscesses $[1,2]$. Tumor ne- 


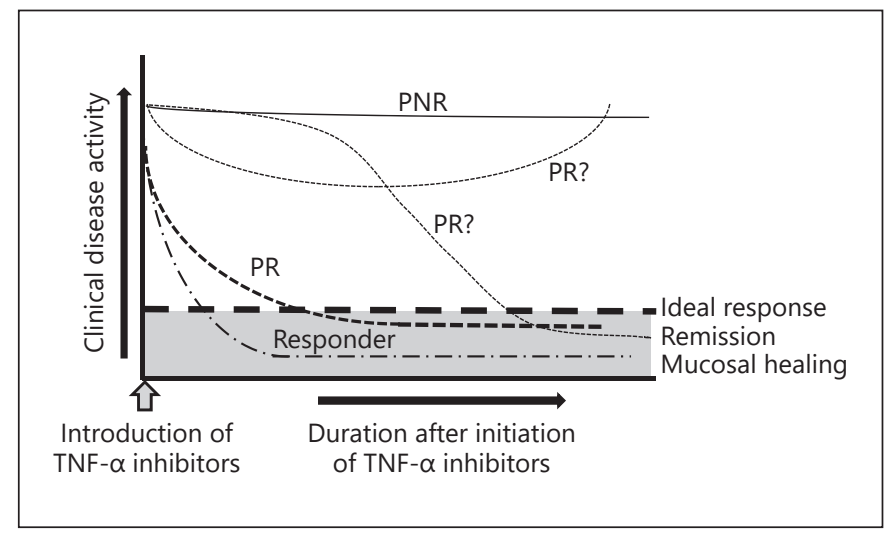

Fig. 1. Concept of primary nonresponders (PNR) and partial responders (PR) after the induction of TNF- $\alpha$ inhibitors.

crosis factor- $\alpha$ (TNF- $\alpha$ ) inhibitors, such as infliximab (IFX) and adalimumab (ADA), improve treatment efficacy and long-term outcomes in patients with CD. Although TNF- $\alpha$ inhibitors are highly effective in patients with $\mathrm{CD}[3,4]$, there are primary nonresponders (PNR), who do not respond at all to induction therapy with TNF- $\alpha$ inhibitors. In randomized controlled trials and cohort studies of IFX for patients with CD, primary nonresponse rates were $8-42 \%$ and primary nonremission rates were $30-52 \%[5,6]$. Thus, in clinical settings, there are partial responders (PR) categorized as neither PNR nor responders, who display a partial response without satisfying effects in the induction phase of TNF- $\alpha$ inhibitors. Primary treatment failure of TNF- $\alpha$ inhibitors can be associated with poor long-term outcomes, including a high risk of surgery or sustained active disease despite medical interventions [7]. Patients who failed to obtain an ideal initial response show poor outcome, while Buhl et al. [8] reported that the population with deep remission increased to $25 \%$ at 2 years after IFX initiation among patients who had partial response after 1 year of IFX treatment. These results suggested that the initial response to TNF- $\alpha$ inhibitors can be associated with long-term outcomes, but some patients may show good long-term outcomes even when the initial response to TNF- $\alpha$ inhibitors was partial (Fig. 1). Thus, management of PR is important to improve the long-term efficacy.

To date, the definitions of PNR and PR are different among studies and not well established. In addition, the treatment strategy for PNR or PR is not well discussed. In order to minimize the populations of PNR and PR, it is important to optimize the usage of TNF- $\alpha$ inhibitors
Table 1. Issues discussed regarding $\mathrm{PNR}$ and $\mathrm{PR}$ in this meeting

1. Definition and management of PNR

1.1 Definition of PNR

1.2 Management of PNR

2. Definition and clinical management of $P R$

2.1 Definition of PR

2.2 Proportion of PR who started infliximab and their clinical background

2.3 Management of PR

2.4 Management of patients who are still PR after treatment intensification

2.5 PR prevention

PNR, primary nonresponders; PR, partial responders.

and concomitant medications. It may also be important to select the patient populations and timing of judging efficacy for TNF- $\alpha$ inhibitors. In this report, we summarized the expert opinions from the Japan Round Table Discussion on IBD (JRT) Meeting, which was attended by 36 Japanese IBD experts. Here, we characterized the PNR and PR to TNF- $\alpha$ inhibitors among the CD population and discussed the factors influencing the efficacy of induction therapy with TNF- $\alpha$ inhibitors. We also discussed how to manage PNR and PR to TNF- $\alpha$ inhibitors.

\section{Process of the Round-Table Meeting}

Thirty-six Japanese IBD experts attended the JRT held in Tokyo, Japan, on August 25, 2018. Before starting the group discussion, a short review lecture (30 min) was given about the proposed concept of PNR and PR and current information regarding the factors associated with therapeutic responses to TNF- $\alpha$ inhibitors as described in the Introduction [1-8]. Participants attended the round-table group discussion in 4 different groups (110 min) regarding the 7 issues related to PNR and PR, which were determined by the core members of this meeting (Table 1). In each group discussion, there was 1 modulator in each group, and the opinions of the attendees ( 8 attendees in each group without organizers) were accumulated. After completing the group discussion, opinions and comments from each group were presented. These opinions were discussed by all participants and were summarized by the chairman and attendees $(80 \mathrm{~min})$. 
Table 2. Management of PNR to TNF- $\alpha$ inhibitors

\begin{tabular}{lllll}
\hline Management & $\begin{array}{l}\text { Group 1 } \\
(n=8)\end{array}$ & $\begin{array}{l}\text { Group 2 } \\
(n=8)\end{array}$ & $\begin{array}{l}\text { Group 3 } \\
(n=8)\end{array}$ & $\begin{array}{l}\text { Group } 4 \\
(n=8)\end{array}$ \\
\hline $\begin{array}{l}\text { Reassessment of intestinal complications } \\
\text { Switch TNF- } \alpha \text { inhibitor to another TNF- } \alpha \text { inhibitor }\end{array}$ & 8 & 8 & 8 & 8 \\
Switch TNF- $\alpha$ inhibitor to ustekinumab & 3 & - & 7 & 8 \\
Dose optimization of TNF- $\alpha$ inhibitors & - & - & 2 & - \\
Addition or dose optimization of immunomodulators & - & - & 5 & 8 \\
Hospitalization with total parental nutritional therapy & 8 & - & - & 8 \\
Addition of enteral nutrition therapy & 8 & - & - & - \\
Addition of metronidazole & $1^{\mathrm{b}}$ & - & - & - \\
\hline
\end{tabular}

Number of participants who agreed to the proposal for the management of PNR is shown. PNR, primary nonresponders; TNF- $\alpha$; tumor necrosis factor- $\alpha$; -, not discussed. ${ }^{a}$ In cases of mild disease activity. ${ }^{b}$ In cases with colonic lesions.

\section{Summary of the Meeting}

\section{Definition of PNR to TNF- $\alpha$ Inhibitors}

PNR after the initiation of TNF- $\alpha$ inhibitors need to be assessed early due to their poor prognosis [7]. However, the response rate can gradually increase from the first to the third administration of TNF- $\alpha$ inhibitors $[4,9]$. Therefore, it is premature to evaluate PNR soon after the first injection, as more accurate evaluations are made after the third injection. Thus, PNR are patients in whom clinical symptoms and serum C-reactive protein (CRP) levels do not improve at all or worsen after 3 injections of TNF- $\alpha$ inhibitors ( $8-10$ weeks after initial IFX administration or 6-8 weeks after initial ADA administration). Intestinal complications (e.g., stenosis, internal fistula, abscess, and perianal disease) are risk factors for PNR [10, 11].

\section{Management of PNR to TNF- $\alpha$ Inhibitors}

Intestinal complications, such as stenosis, internal fistula, and abscess, can develop during the course of CD even if they were nonexistent at the time of diagnosis. Patients need to be (re-)evaluated for intestinal complications by using imaging modalities when they are suspected to be PNR. If complications, such as severe strictures, fistulas, and abscesses, are detected during examination, surgery should be performed if applicable. The experts felt that primary nonresponse was rare when severe intestinal complications were absent as some extent of efficacy can usually be observed in patients with $\mathrm{CD}$ in the absence of intestinal complications. Intestinal complications are usually assessed by imaging modalities before starting TNF- $\alpha$ inhibitors, and these patients would not be treated with TNF- $\alpha$ inhibitors in the clinical practice of Japanese experts. Therefore, it was difficult to reach a consensus due to the limited experience of PNR management. For PNR without apparent intestinal complications that require treatment, medical treatment options, such as switching TNF- $\alpha$ inhibitors, proposed in the discussion of each group are listed in Table 2.

\section{Definition of PR to TNF- $\alpha$ Inhibitors}

$\mathrm{PR}$ are patients in whom some therapeutic effects are observed but clinical remission is not achieved by induction therapy with TNF- $\alpha$ inhibitors. If the patients meet one of the following conditions after 3 injections of TNF- $\alpha$ inhibitors (8-10 weeks after initial IFX administration or 6-8 weeks after initial ADA administration), they can be classified as PR: (1) both serum CRP levels and clinical symptoms decreased or improved, but did not become negative or disappear; (2) clinical symptoms were absent, but serum CRP levels were positive; and (3) even if patients' symptoms were absent and serum CRP levels were negative, active inflammation was observed on imaging (e.g., computed tomography [CT], magnetic resonance imaging [MRI], and ileocolonoscopy). Clinical activity can be evaluated using a quantitative clinical activity index, such as Crohn's disease activity index (CDAI), but the calculation of CDAI is time consuming and not clinically practical. Therefore, clinical activity is usually determined by the patients' symptoms assessed by the physicians and blood tests, such as serum CRP levels. In particular, when patients have clinical symptoms, it is necessary to evaluate if symptoms are caused by the active intestinal inflammation of CD or not. For that purpose, intestinal disease activity should be assessed using imaging modalities such as CT and MRI. In addition, serum
Iijima et al. 
albumin and hemoglobin levels are useful for evaluating PR since these blood markers reflect the nutritional and inflammatory status. PR can be determined by endoscopy (ileocolonoscopy, capsule endoscopy, and balloon-assisted enteroscopy) in the absence of clinical symptoms and elevated CRP levels. In such cases, the optimal timing to evaluate PR for mucosal lesions has not yet been determined.

\section{Proportion of PR to TNF- $\alpha$ Inhibitors and Their Clinical Background}

The experts felt that the proportion of $\mathrm{PR}$ after the initiation of TNF- $\alpha$ inhibitors was approximately 20 $30 \%$ based on their clinical experience. Patients with extensive small intestinal lesions, peri-anal lesions, and intestinal complications, such as strictures and fistulas, may be prone to becoming PR as they are considered to be resistant to TNF- $\alpha$ inhibitors compared to those without these lesions. Intensification of treatment may not always be necessary for all PR, and the population who required intensification was discussed. As a result, if CRP levels and clinical symptoms do not improve or worsen at the time of the fourth injection (10-14 weeks after IFX initiation or 6-8 weeks after ADA initiation) compared to the prior injection, treatment needs to be intensified. If clinical symptoms and CRP levels improve from the prior administration, treatment intensification is not necessary. However, in such cases, the condition of the patient should be observed carefully. The proportion of PR who requires treatment intensification was estimated to be about $50-60 \%$, based on the experience of the experts.

\section{Management of PR to TNF- $\alpha$ Inhibitors}

Low serum drug concentrations of TNF- $\alpha$ inhibitors can be a factor for PR. Dose optimization needs to be performed to increase the concentration of TNF- $\alpha$ inhibitors. Dose optimization of IFX can include not only increasing the dose of IFX to $10 \mathrm{mg} / \mathrm{kg}$ in 8 -week intervals, but also shortening the administration interval to 4 weeks. For cases of ADA treatment with $40 \mathrm{mg}$ biweekly, the dose should be increased to $80 \mathrm{mg}$ biweekly or shortened to $40 \mathrm{mg}$ weekly, although weekly $40 \mathrm{mg}$ injection of ADA is not approved in Japan. Some experts experienced that altering IFX injection to $5 \mathrm{mg} / \mathrm{kg}$ with 4 -week intervals was sometimes effective in patients initially administered $10 \mathrm{mg} / \mathrm{kg}$ IFX at 8-week intervals. Shortening of the interval can be more effective than increasing the dose because the serum concentration of TNF- $\alpha$ inhibitors is maintained at stably high levels [12-14]. Although dose

Primary Nonresponders and Partial

Responders to Anti-TNF Biologics optimization based on the serum trough level is ideal, it is not covered by Japanese national health insurance and can only be measured in limited institutions. In addition, the dose and timing of TNF- $\alpha$ inhibitor injections cannot be flexibly changed in Japan. In patients who are not taking immunomodulators (IM), it is worth considering the addition of IM at the time of TNF- $\alpha$ inhibitor dose optimization. Before using IM, risks of IM need to be estimated by the examination of nudix hydrolase 15 (NUDT15) genetic polymorphisms in order to screen high-risk patients for severe side effects, such as severe leukopenia and alopecia [15].

Management of Patients Who Are Still PR after Dose Intensification of TNF- $\alpha$ Inhibitors

When dose optimization of TNF- $\alpha$ inhibitors does not result in the improvement of clinical activity, switching between TNF- $\alpha$ inhibitors with the same mechanism of action is not expected to have high efficacy. In such cases, it will be better to switch to a drug with a different mechanism of action, such as ustekinum$\mathrm{ab}$, which inhibits the interleukin-12/23 p40 subunit. Using vedolizumab may also be an option, but it was not approved for use at the time of the meeting. The addition of enteral nutritional therapy or cytapheresis (in cases with colonic involvement) can also be considered, but evidence is scarce, and their efficacy may not be sufficient.

\section{Interventions and Pretreatments to Prevent $P R$ for TNF- $\alpha$ Inhibitors}

As smoking is a significant risk factor for worsening disease activity of $\mathrm{CD}$ and induces unresponsiveness to drug treatment $[16,17]$, we need to instruct patients to stop smoking if the patient is a smoker. Previous studies have shown the benefit of using IM in combination with TNF- $\alpha$ inhibitors to prevent the development of antidrug antibodies [3]. Therefore, it would be better to use concomitant IM at the initiation of TNF- $\alpha$ inhibitors, especially when using a chimeric antibody, such as IFX. In cases of severe disease activity, it may be effective to conduct total parental nutrition therapy (TPN) for a certain period before the initiation of TNF- $\alpha$ inhibitors in order to reduce intestinal inflammation. Some experts perform TPN in very severe cases and believe it is effective, but it is sometimes difficult to obtain the patients' consent to perform TPN with long-term hospitalization. Therefore, more evidence needs to be accumulated to determine the efficacy of combinatorial therapies. 


\section{Discussion/Conclusion}

The Japanese IBD experts discussed about the evaluation and management of PNR and PR after the initiation of TNF- $\alpha$ inhibitors. The discussion described in this report was mostly based on the daily clinical practice of experts working in major Japanese high-volume IBD centers but not based on high-level evidence. We did neither propose a systematic list of questions nor use methodologies for qualitative data collection, which are used to represent a meta-analysis or a guideline based on a strict process. However, the content can be useful information for the consideration of treatment strategies when physicians do not achieve ideal outcomes, such as clinical remission and mucosal healing, after the initiation of TNF- $\alpha$ inhibitors. More evidence needs to be accumulated in order to improve the efficacy of TNF- $\alpha$ inhibitors to prevent and manage PNR and PR.

\section{Appendix}

JRT Group

Hiroshi Araki (Gifu University), Toshimitsu Araki (Mie University), Katsuyuki Fukuda (St. Luke's International Hospital), Kitaro Futami (Fukuoka University Chikushi Hospital), Nobuyuki Hida (Hyogo College of Medicine), Sakiko Hiraoka (Okayama University), Tadakazu Hisamatsu (Kyorin University School of Medicine), Ryota Hokari (National Defense Medical College), Hiroki Ikeuchi (Hyogo College of Medicine), Tetsuya Ishida (Ishida Clinic of IBD and Gastroenterology), You Ishiguro (Hirosaki National Hospital), Jun Kato (Mitsui Memorial Hospital), Shingo Kato (Saitama Medical Center, Saitama Medical University), Takehiko Katsurada (Hokkaido University), Yutaka Kougo (International University of Health and Welfare Hospital), Masaaki Matsukawa (Showa University Toyosu Hospital), Takayuki Matsumoto (Iwate Medical University), Shiro Nakamura (Hyogo College of Medicine), Hiroshi Nakase (Sapporo Medical University), Masayuki Saruta (The Jikei University School of Medicine), Ken Sugimoto (Hamamatsu University School of Medicine), Yoshiaki Takeuchi (Showa University Hospital), Ken Takeuchi (Toho University Sakura Medical Center), Torao Tanaka (Maeda Hospital), Motoi Uchino (Hyogo College of Medicine), Kenji Watanabe (Hyogo College of Medicine), Kaoru Yokoyama (Kitasato University), and Naoki Yoshimura (Tokyo Yamate Medical Center).

\section{Acknowledgement}

We thank all the members of the JRT Group for the fruitful discussions. All authors contributed significantly to this article or and approved the final manuscript.

\section{Statement of Ethics}

The authors have no ethical conflicts to disclose. This study was a report of a IBD specialist meeting not related to either a study using human data and samples or animal study which needs approval from the ethical committee.

\section{Disclosure Statement}

H.I. received lecture fees from Mitsubishi Tanabe Pharma, Ab$\mathrm{bVie}$, Janssen, EA Pharma, and Eisai and research grants from AbbVie and Nippon Kayaku. T.K. received consultancy fees from AbbVie GK, Alfresa Pharma, Covidien, Eli Lilly, Ferring Pharmaceuticals, Janssen, Kyorin Pharmaceutical Co., Ltd., Mochida Pharmaceutical, Nippon Kayaku, Pfizer, Takeda Pharmaceutical, and Thermo Scientific, lecture fees from AbbVie GK, Ajinomoto Pharma, Asahi Kasei Medical, Astellas, Alfresa Pharma, Celltrion, EA Pharma Co., Ltd., Kyorin Pharmaceutical Co., Ltd., Nippon Kayaku, Mochida Pharmaceutical, Takeda Pharmaceutical, Mitsubishi Tanabe Pharma, ZERIA, Eisai Co., Ltd., Gilead Sciences, Janssen, and JIMRO Co., Ltd., and research funding from AbbVie GK, EA Pharma Co., Ltd., Otsuka Holdings Co., Ltd., ZERIA, Kyorin Pharmaceutical Co., Ltd., Mochida Pharmaceutical, EA Pharma Co., Ltd., Thermo Fisher Scientific, Alfresa Pharma, Nippon Kayaku, and Asahi Kasei Medical. K.K. received lecture fees from Mitsubishi Tanabe Pharma, AbbVie, Janssen, and Takeda Pharmaceutical Co. and a research grant from AbbVie. S.S. received lecture fees from Mitsubishi Tanabe Pharma, AbbVie, Janssen, EA Pharma, and Eisai. Y.S. received lecture fees from AbbVie, Mitsubishi Tanabe Pharma, Zeria, Mochida Pharmaceutical Co., Ltd., Kyorin Pharmaceutical Co., Ltd., and Janssen and has received research grants from AbbVie, Mitsubishi Tanabe Pharma, EA Pharma Co., Ltd., JIMRO Co., Ltd., Mochida Pharmaceutical Co., Ltd., and Kissei. M.W. received lecture fees from Mitsubishi Tanabe Pharma Corporation, Takeda Pharmaceutical Co., Ltd., Zeria Pharmaceutical Co., Ltd., Astellas Pharma Inc., Nippon Kayaku Co., Ltd., Mochida Pharmaceutical Co., Ltd., Pfizer Japan Inc., Janssen Pharmaceutical KK, Kissei Pharmaceutical Co., Ltd., Celltrion Healthcare Co., Ltd., Celgene KK, and Gilead Sciences, Inc., consultancy fees from Takeda Pharmaceutical Co., Ltd., AbbVie GK, EA Pharma Co., Ltd., Eli Lilly Japan KK, Gilead Sciences, Inc., and research funding from Mitsubishi Tanabe Pharma Corporation, Takeda Pharmaceutical Co., Ltd., Zeria Pharmaceutical Co., Ltd., Astellas Pharma Inc., MSD KK, Daiichi Sankyo Co., Ltd., Taiho Pharmaceutical Co., Ltd., Nippon Kayaku Co., Ltd., Mochida Pharmaceutical Co., Ltd., Ayumi Pharmaceutical Corporation, Miyarisan Pharmaceutical Co., Ltd., JIMRO Co., Ltd., Asahi Kasei Medical Co., Ltd., Kyorin Pharmaceutical Co., Ltd., AbbVie GK, Kyowa Hakko Kirin Co., Ltd., EA Pharma Co., Ltd., Pfizer Japan Inc., Kissei Pharmaceutical Co., Ltd., Kaken Pharmaceutical Co., Ltd., Alfresa Pharma Corporation, Toray Industries, Inc., Chugai Pharmaceutical Co., Ltd., Gilead Sciences, Inc., and Fujirebio Inc. T.H. received lecture fees from Aspen Japan KK, AbbVie GK, Ferring, Gilead Sciences, Janssen, JIMRO, Kisse Pharmaceutical, Mitsubishi-Tanabe Pharma, Mochida Pharmaceutical, Nippon Kayaku Pfizer, Takeda Pharmaceutical, and Zeria Pharmaceutical, advisory/consultancy fees from AbbVie, Bristol-Myers Squibb, Celltrion, EA Pharma, Eli Lilly, Gilead Sciences, Janssen, Kyorin,
Iijima et al. 
Mitsubishi-Tanabe Pharma, Nichi-Iko Pharmaceutical, Pfizer, Takeda Pharmaceutical, and Zeria Pharmaceutical, and research grants from AbbVie, EA Pharma, JIMRO, Otuska Holdings, and Zeria Pharmaceuticals.

\section{Funding Sources}

Mitsubishi Tanabe Pharma (Osaka, Japan) provided the meeting expenses and travel expenses for the participants.

\section{Author Contributions}

H.I. was involved in the drafting and critical revision of the manuscript. T.K., M.N., S.S., K.K., Y.S., M.W., and T.H. critically revised the manuscript for important intellectual content. All authors had major roles in the round table discussion and approved the final manuscript.

\section{References}

1 Verstockt B, Ferrante M, Vermeire S, Van Assche G. New treatment options for inflammatory bowel diseases. J Gastroenterol. 2018 May;53(5):585-90.

2 Matsuoka K, Kobayashi T, Ueno F, Matsui T, Hirai F, Inoue N, et al. Evidence-based clinical practice guidelines for inflammatory bowel disease. J Gastroenterol. 2018 Mar;53(3):30553.

3 Colombel JF, Sandborn WJ, Reinisch W, Mantzaris GJ, Kornbluth A, Rachmilewitz D, et al.; SONIC Study Group. Infliximab, azathioprine, or combination therapy for Crohn's disease. N Engl J Med. 2010 Apr; 362(15):1383-95.

4 Hanauer SB, Feagan BG, Lichtenstein GR, Mayer LF, Schreiber S, Colombel JF, et al.; ACCENT I Study Group. Maintenance infliximab for Crohn's disease: the ACCENT I randomised trial. Lancet. 2002 May;359(9317): 1541-9.

5 Papamichael K, Van Stappen T, Jairath V, Gecse K, Khanna R, D'Haens G, et al. Review article: pharmacological aspects of anti-TNF biosimilars in inflammatory bowel diseases. Aliment Pharmacol Ther. 2015 Nov;42(10): 1158-69.

6 Ricart E, Panaccione R, Loftus EV, Tremaine WJ, Sandborn WJ. Infliximab for Crohn's disease in clinical practice at the Mayo Clinic: the first 100 patients. Am J Gastroenterol. 2001 Mar;96(3):722-9.
7 Buhl S, Steenholdt C, Rasmussen M, Borghede MK, Brynskov J, Thomsen OO, et al. Outcomes After Primary Infliximab Treatment Failure in Inflammatory Bowel Disease. Inflamm Bowel Dis. 2017 Jul;23(7):1210-7.

8 Buhl S, Kristina Borghede M, Brynskov J, Steenholdt C, Rasmussen M, Andrew Ainsworth M. Outcome of continued infliximab therapy in Crohn's disease patients with response but without remission after one year of infliximab - a retrospective cohort study. Scand J Gastroenterol. 2018 Aug;53(8):930-7.

9 Targan SR, Hanauer SB, van Deventer SJ, Mayer L, Present DH, Braakman T, et al. A shortterm study of chimeric monoclonal antibody cA2 to tumor necrosis factor alpha for Crohn's disease. Crohn's Disease cA2 Study Group. N Engl J Med. 1997 Oct;337(15):1029-35.

10 Papamichael K, Gils A, Rutgeerts P, Levesque BG, Vermeire S, Sandborn WJ, et al. Role for therapeutic drug monitoring during induction therapy with TNF antagonists in IBD: evolution in the definition and management of primary nonresponse. Inflamm Bowel Dis. 2015 Jan;21(1):182-97.

11 Ding NS, Hart A, De Cruz P. Systematic review: predicting and optimising response to anti-TNF therapy in Crohn's disease - algorithm for practical management. Aliment Pharmacol Ther. 2016 Jan;43(1):30-51.

12 Hibi T, Sakuraba A, Watanabe M, Motoya S, Ito $\mathrm{H}$, Motegi $\mathrm{K}$, et al. Retrieval of serum infliximab level by shortening the maintenance infusion interval is correlated with clinical efficacy in Crohn's disease. Inflamm Bowel Dis. 2012 Aug;18(8):1480-7.
13 Suzuki $\mathrm{Y}$, Matsui T, Ito $\mathrm{H}$, Ashida T, Nakamura S, Motoya S, et al. Circulating Interleukin 6 and Albumin, and Infliximab Levels Are Good Predictors of Recovering Efficacy After Dose Escalation Infliximab Therapy in Patients with Loss of Response to Treatment for Crohn's Disease: A Prospective Clinical Trial. Inflamm Bowel Dis. 2015 Sep;21(9):2114-22.

14 Hofmekler T, Bertha M, McCracken C, Martineau B, McKinnon E, Schoen BT, et al. Infliximab Optimization Based on Therapeutic Drug Monitoring in Pediatric Inflammatory Bowel Disease. J Pediatr Gastroenterol Nutr. 2017 Apr;64(4):580-5.

15 Yang SK, Hong M, Baek J, Choi H, Zhao W, Jung Y, et al. A common missense variant in NUDT15 confers susceptibility to thiopurine-induced leukopenia. Nat Genet. 2014 Sep;46(9):1017-20.

16 Barber GE, Yajnik V, Khalili H, Giallourakis C, Garber J, Xavier R, et al. Genetic Markers Predict Primary Non-Response and Durable Response To Anti-TNF Biologic Therapies in Crohn's Disease. Am J Gastroenterol. 2016 Dec;111(12):1816-22.

17 Yoon SM, Haritunians T, Chhina S, Liu Z, Yang S, Landers C, et al. Colonic Phenotypes Are Associated with Poorer Response to AntiTNF Therapies in Patients with IBD. Inflamm Bowel Dis. 2017 Aug;23(8):1382-93. 